\title{
LUXE: \\ COMBINING HIGH ENERGY AND INTENSITY TO SPARK THE VACUUM
}

- Beate Heinemann ${ }^{1}$, Tom Heinzl ${ }^{2}$ and Andreas Ringwald ${ }^{3}$ - https://doi.org/10.1051/epn/2020401

- ${ }^{1}$ DESY and Albert-Ludwigs-Universität Freiburg $-{ }^{2}$ University of Plymouth, UK $-{ }^{3}$ DESY

The vacuum of quantum electrodynamics (QED) can be viewed as a medium akin to a dielectric which can be polarised by external fields. If these are sufficiently strong, the response of the vacuum becomes nonlinear and involves phenomena such as lightby-light scattering ('nonlinear optics') and, in extremis, 'dielectric breakdown', i.e. real pair production, if a critical field strength is exceeded. The LUXE experiment aims to realise near-critical fields through collisions of photons stemming from an ultra-intense optical laser with high energy electrons or photons provided by the European XFEL linear accelerator. This set-up provides a golden opportunity to enter the uncharted territory of strong-field quantum electrodynamics in the non-perturbative regime. 


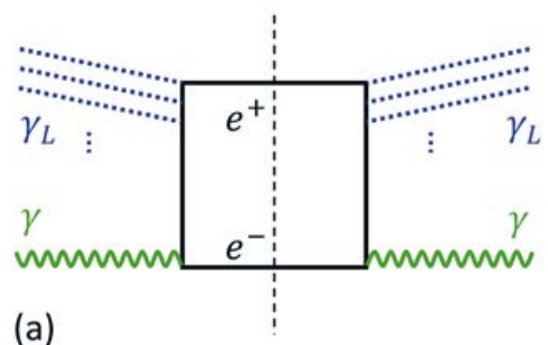

(a)

\section{The quantum vacuum}

\section{and its breakdown}

Faraday, Maxwell and Hertz have told us that the vacuum is filled with electromagnetic fields, but it took an Einstein to realise that this does not require a luminiferous aether: Fields and their wave excitations do not need a medium in which to propagate. Ironically, with the advent of quantum field theory, the idea of the vacuum as a medium was resurrected. It is the quantum fields themselves, through their virtual fluctuations, which lend the vacuum properties akin to a dielectric [1]. The basic phenomenon in this context is vacuum polarisation: Applying a weak external field (e.g. due to an electron), the vacuum dipoles formed by virtual electron positron pairs align and screen the electron charge. A probe particle 'diving' into the polarisation cloud surrounding the electron will thus 'see' the elementary charge, $e$, increasing with probe energy (charge renormalisation). As a result, 'perturbative QED', based on the coupling being small $\left(e^{2} / 4 \pi<1\right)$ becomes invalid at ultra-high energies.

Alternatively, one may ask what happens if one applies stronger and stronger external fields. The first issue to address is the notion of a 'strong' field in QED. As a relativistic quantum field theory, QED contains two fundamental constants of nature, Planck's constant, $\hbar$, and the speed of light, $c$. Combining these with the QED parameters, $e$ and $m$, the electron mass, one can form the QED electric field strength, $E_{S}=m^{2} c^{3} / e \hbar=1.3 \times 10^{18} \mathrm{~V} / \mathrm{m}$. This enormous field magnitude is typical for elementary processes in QED. The associated energy balance, $e E_{S}(\hbar / m c)=m c^{2}$, corresponds to an energy transfer of $m c^{2}$ (the electron rest energy) over the distance of a Compton wavelength, $\hbar / m c$. The challenge, though, is to realise the QED field strength $E_{S}$ over macroscopic distances, say $1 \mu \mathrm{m}$ or larger. Sauter noted in 1931 that even in this classical field scenario the anti-particles of the electrons, the positrons, become relevant [2]. This was further elaborated by Schwinger (1951) who interpreted the appearance of the positrons as an instability of the QED vacuum which starts to 'spark' with electron positron pairs [3]. The vacuum thus becomes 'conducting' similarly to an insulator suffering dielectric breakdown.

Pictorially, the situation is described in Fig.1(a) which displays a vacuum polarisation loop forming a virtual electron positron dipole. This dipole is exposed to the superimposed fields of laser and probe photons, $\gamma_{L}$ and

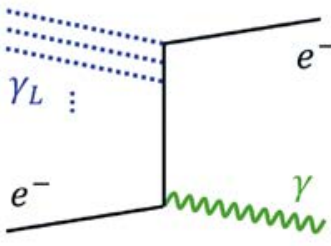

(c) $\gamma$, respectively. This diagram describes the nonlinear interaction of light with light, a subtle quantum effect first analysed in [1]. Schwinger calculated its imaginary part, which physically amounts to pair production via the nonlinear Breit-Wheeler process, $\gamma+n \gamma_{L} \rightarrow e^{+} e^{-}$, see Fig.1(b), and corresponds to the creation of matter from light.

The regime of electromagnetic field strengths reaching or even exceeding $E_{S}$ is referred to as strong-field QED. It may be realised by approaching highly localised charge distributions, for instance at a distance of $30 \mathrm{fm}$ from an electron. Alternatively, one may probe bound Coulomb systems by colliding heavy ions or by analysing the spectroscopy of heavy atoms, hence studying the physics at large atomic number $Z$ [4]. High field strengths are also relevant for the early Universe and several astrophysical phenomena such as the gravitational collapse of Black Holes [5], the propagation of cosmic rays [6], or the surface of strongly magnetised neutron stars [7], and for future linear high energy $e^{+} e^{-}$-colliders [8]. Unfortunately, most suggestions above suffer either from nuclear effects (at large Z) or experimental inaccessibility (astrophysics).

\section{The LUXE experiment}

To overcome these problems, the LUXE collaboration proposes [9] to approach the critical field, $E_{\varsigma}$, by exploiting recent advances in high-power laser technology and the 'magic' of Lorentz invariance. The increase in laser intensity due to chirped pulse amplification (Nobel prize 2018 [10]), allows production of optical-laser fields with a lab-measured strength just a few orders of magnitude below the Schwinger field. Employing relativistic electrons $\left(E_{e}>5 \mathrm{GeV}\right)$ a gamma factor $\gamma_{e}>10^{4}$ is reached, so that even at lower laser intensities the electron sees the critical field in its rest frame.

The basic experimental ideas are the following: one can send the electrons through a tungsten converter foil, in order to generate photons $(\gamma)$ by bremsstrahlung and then collide these with the photons $\left(\gamma_{L}\right)$ of the laser beam, $\gamma+n \gamma_{L} \rightarrow e^{+} e^{-}$, see Fig. 1 (b). Alternatively, one may collide the electrons directly with the laser beam, $e^{-}+n \gamma_{L} \rightarrow e^{-}+\gamma$, see Fig. 1 (c).

A previous experiment (SLAC E144) has employed $50 \mathrm{GeV}$ electrons and a $1 \mathrm{TW}$ laser to explore what is now called two-step trident pair production. This is a succession of the two nonlinear processes introduced above,
4FIG. 1:

Diagrams representing basic processes of strong-field QED. (a) Light-by-light scattering of laser photons $\left(\gamma_{\mathrm{L}}\right)$ and probe photons ( $\gamma)$. (b) Nonlinear

Breit-Wheeler pair production. (c) Nonlinear Compton scattering. 
v FIG. 2:

Sketch of the experimental setup for the $\gamma_{B}$-laser collisions. A dipole magnet and a set of detectors (pixel tracker, calorimeters) for the $\mathrm{e}^{ \pm}$and $\mathrm{y}$ detection are shown behind the IP. $A \gamma_{B}-$ monitoring system after the tungsten foil is also shown. using the photons produced in nonlinear Compton scattering by colliding them with the laser photons to produce pairs, recall Fig.1. The observed production rates were proportional to higher-than-linear powers of the laser intensity thus clearly detecting nonlinearity. The goal of LUXE is to enter far deeper into the strong-field region by using the much more powerful lasers available today, together with the high energy electrons from the European XFEL (EuXFEL) accelerator. This will enable probing the transition from the nonlinear to the non-perturbative regime, which may be characterised by a key parameter, the dimensionless laser amplitude, $\xi$. It is defined as the ratio of the work done by the laser field $E_{L}$ when 'pushing' an electron across a reduced laser wavelength and the electron rest energy. When this is of order unity, an electron probing the laser becomes relativistic. There is more to this parameter, though: A process involving $n>1$ laser photons (see again Fig. 1 ) contributes with a probability amplitude proportional to $\xi^{n}$. When $\xi$ approaches unity, all these $n$-photon amplitudes become equally important, and one has to sum over all of them. As higher order effects thus cease to be 'small perturbations' of lower order ones, the overall process becomes non-perturbative. The SLAC E144 experiment had $\xi \sim 0.5$, still in the perturbative regime. For LUXE we expect $\xi>2$, which takes us well into the uncharted non-perturbative regime.

The second key parameter is the quantum parameter, $\chi_{e} \equiv 2 \gamma_{e} E_{L} / E_{S}(1+\cos \theta)$, where $\theta$ is the angle between the laser and particle beams. For LUXE, with $\gamma_{e}=3 \times 10^{4}$ from the EuXFEL beam, the genuine quantum regime, $\chi_{e}>1$, is reached for $\xi>5$.

LUXE is also in the unique position to test the structure of the vacuum near the Schwinger limit via light-bylight scattering. High-energy probe photons, $\gamma_{B}$, created through bremsstrahlung will be brought into collision with the ultra-intense laser-beam. This will allow to

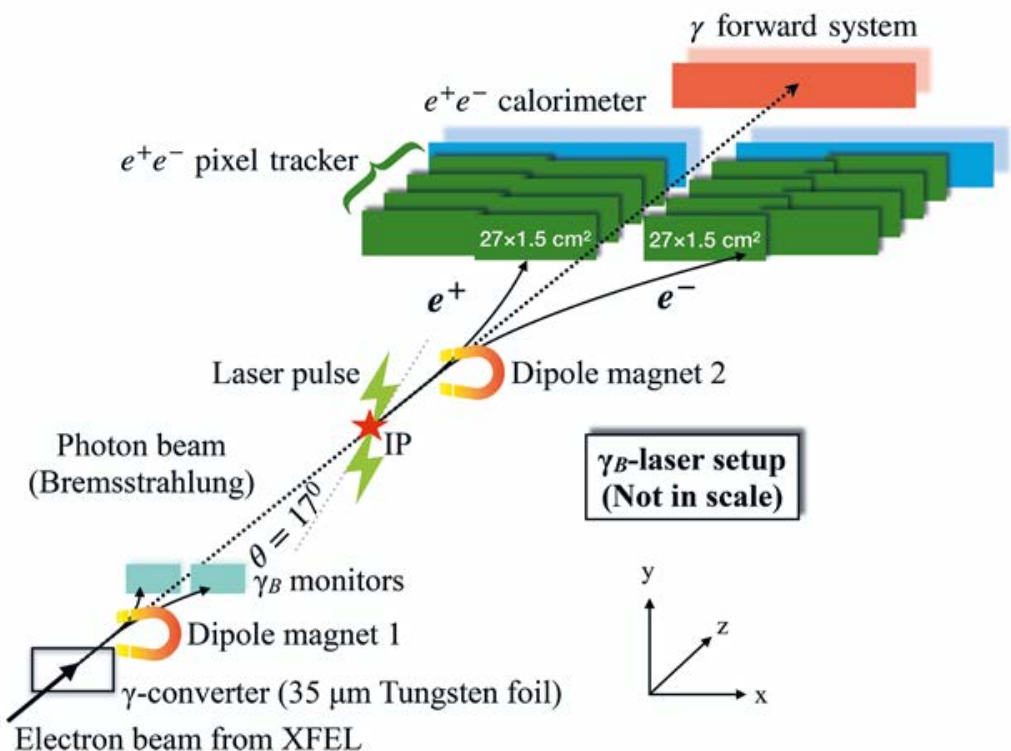

study the nonlinear Breit-Wheeler process in isolation for the first time. The relevant parameter in this case is $\chi_{\gamma}=\xi \times \omega_{L} \omega_{B}\left(\hbar / m c^{2}\right)^{2}, \omega_{\mathrm{L}}$ and $\omega_{\mathrm{B}}$ denoting the photon frequencies. At low $\xi$ and $\chi_{\gamma}$, the rate follows the power-law $\left(\sim \xi^{2 \mathrm{n}}\right)$ based on counting the number of photons contributing in perturbation theory. In contrast, when $\xi \gg 1$ and $\chi_{\gamma} \approx 1$, a non-perturbative QED calculation shows that the rate of $e^{+} e^{-}$production is proportional to $\chi_{\gamma} \exp \left(-8 / 3 \chi_{\gamma}\right)$. Measuring this rate will be a direct experimental test of non-perturbative QED.

\section{Experimental setup}

The EuXFEL is designed to run with energies up to $E_{\mathrm{e}}=17.5 \mathrm{GeV}$ and trains of 2700 electron bunches, each typically containing $1.5 \times 10^{9}$ electrons that pass at a rate of $10 \mathrm{~Hz}$. For the beam-laser interaction, one electron bunch per bunch train is extracted using a fast kicker magnet and guided to the interaction region hosted in a currently unused annex of the shaft at the end of the linear accelerator.

The laser envisaged has a power of $40 \mathrm{TW}$ at an initial stage and about $400 \mathrm{TW}$ in a later stage. Its light will be focused to achieve intensities of about $10^{20} \mathrm{~W} / \mathrm{cm}^{2}$ initially (and $10^{21} \mathrm{~W} / \mathrm{cm}^{2}$ later). An elaborate laser diagnostics system will be designed to measure the absolute laser intensity to better than $5 \%$. The angle between the beam and the laser will be about $20^{\circ}$.

A schematic layout of the experiment is shown in Fig. 2 for the $\gamma_{B}+n \gamma_{L}$ interactions. For this layout, photons are produced through Bremsstrahlung of the beam electrons on a tungsten foil. For $e+n \gamma_{L}$ interactions (Fig. 1(c)), the layout is similar, except that there is no converter foil, and the particle detection systems are adapted accordingly.

\section{Expected Scientific Results}

Compared to previous, current or planned facilities [12], LUXE will achieve higher values of $\chi_{e}$ for the electron-laser collisions and will be the first to directly study photon-laser collisions. The calculated rate [13] of $e^{+} e^{-}$pairs per laser shot for the $\gamma_{B}+n \gamma_{L}$ process is expected to initially rise like a power-law, however, at $\xi \sim 2$, the rate increase slows down. The goal of LUXE is to measure these rates to better than $10 \%$.

\section{Conclusions}

LUXE will shed new light on the vacuum and reveal new insights into our Universe. It presents a unique opportunity to pioneer a novel regime of quantum physics, the strong-field regime of QED, employing one of the premier European research infrastructures, the European XFEL. The goals are to observe for the first time directly the $\gamma \gamma \rightarrow e^{+} e^{-}$process, and to perform the first studies of the transition from the perturbative to the strong-field (non-perturbative) regime. 


\section{About the Authors}

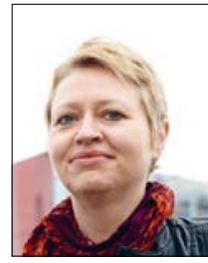

Beate Heinemann is an experimental physicist. She is a leading scientist at DESY and a full professor at the Albert-Ludwigs-Universität Freiburg. Her research interest is the understanding of fundamental laws of Nature that governed the constituents of the Universe in its early phase before stars were formed. She has worked on several large particle physics experiments, most recently the ATLAS experiment at the Large Hadron Collider. She is also leading the LUXE experiment, proposed by Andreas Ringwald, at DESY and the European XFEL to study strong-field QED.

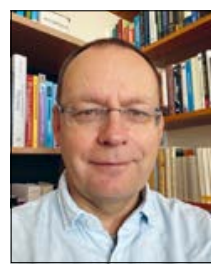

Tom Heinzl is an Associate Professor (Senior Lecturer) in Theoretical Physics at the University of Plymouth, UK. His research interests are strong interactions in various guises, with a current focus on quantum electrodynamics in the presence of ultra-intense laser fields. As a theorist, he is developing (mostly) analytic methods to understand the nonperturbative dynamics involved.

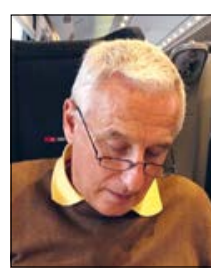

Andreas Ringwald is a theoretical particle physicist at DESY. He is working on a broad class of theoretical, phenomenological and cosmological questions. One focus of this work is on non-perturbative processes in the Standard Model of particle physics. More than a decade ago, he brought up the idea to collide the EU.XFEL beam with an intense laser beam to study non-perturbative pair production in QED.

\section{References}

[1] W. Heisenberg and H. Euler, Z. Phys. 98, 714 (1936).

[2] F. Sauter, Z. Phys. 69, 742 (1931).

[3] J. Schwinger, Phys. Rev. 82, 664 (1951).

[4] I. Pomeranchuk and Y. Smorodinsky, J. Phys. USSR 9, 97 (1945).

[5] R. Ruffini, G. Vereshchagin, and S.-S. Xue, Phys. Rept. 487, 1 (2010).

[6] A. I. Nikishov, Sov. Phys. JETP 14, 393 (1962).

[7] C. Kouveliotou et al., Nature 393, 235 (1998).

[8] M. Bell and J. S. Bell, Part. Accel. 24, 1 (1988).

[9] H. Abramowicz et al., arXiv:1909.00860 (2019).

[10] https://www.nobelprize.org/prizes/physics/2018/ summary/

[12] C. N. Danson et al, High Power Laser Science and Engineering 7, e54 (2019)

[13] A. Hartin, A. Ringwald and N. Tapia, Phys. Rev. D 99, no. 3, 036008 (2019), arXiv:1807.10670.

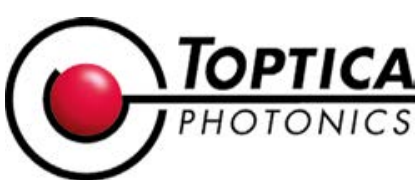

\section{$2500-6900 \mathrm{~cm}^{-1} \mathrm{CW}$ optical parametric oscillator -}

Narrow linewidth, fully-automated tuning for high resolution molecular spectroscopy

T he DLC TOPO is the widest tuning, narrowest linewidth CW OPO. Remote control of all tuning actuators enables hands-free operation over 2500 - 6900 $\mathrm{cm}^{-1}$ without realignment or optics exchanges. The TOPO is the only choice for high-resolution molecular spectroscopy of stretch vibrations.

MIR spectroscopy has never been easier. The powerful TOPO delivers wide tunability, narrow linewidth, and convenient hands-free digital control. There are no modules to exchange and no adjustments to be made by hand. Ease of use and reliable performance make this CW OPO the right choice for challenging applications in MIR spectroscopy and quantum optics:
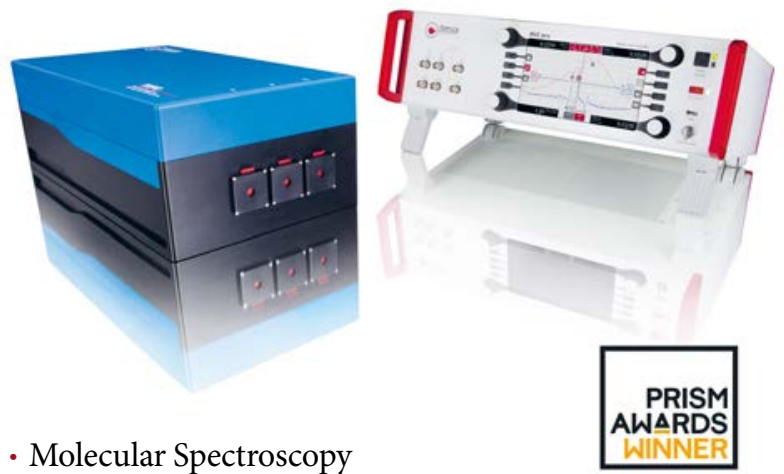

- Molecular Spectroscopy WARDS

- Quantum Optics

- Materials Testing

- Biophotonics

- Physical Chemistry

- Integrated Photonic Devices

The revolutionary TOPO by TOPTICA stands alone as the only fully automated, continuous-wave, singly-resonant optical parametric oscillator laser source on the market. High resolution spectroscopy across $1.45-4.0 \mu \mathrm{m}$ (2500 $6900 \mathrm{~cm}^{-1}$ ) has never been easier.

TOPTICA's unique optical design enables broadly tunable laser light. No module or mirror exchange is necessary. Additionally, the all-digital control electronics enable handsfree coarse tuning, and frequency locking. A wide mode-hopfree tuning range up to $300 \mathrm{GHz}\left(10 \mathrm{~cm}^{-1}\right)$ enables visibility of full spectroscopic signatures. Simultaneously, the TOPO maintains a narrow linewidth $\left(2 \mathrm{MHz}, 1 \cdot 10^{-5} \mathrm{~cm}^{-1}\right)$ giving a solution that reveals narrow atomic and molecular features.

TOPTICA's OPO laser system won the Prism Awards 2019 for scientific lasers at Photonics West!

https://www.toptica.com/topo 